\title{
Double Higgs Production at the LHC as a Robust Test of Little Higgs Models
}

\author{
Claudio O. Dib*, Rogerio Rosenfeld ${ }^{\dagger}$ and Alfonso Zerwekh** \\ *Dept. of Physics, Universidad Técnica Federico Santa María, Valparaíso, Chile \\ ${ }^{\dagger}$ Instituto de Física Teórica - UNESP, Rua Pamplona, 145, 01405-900 São Paulo, SP, Brasil \\ ${ }^{* *}$ Department of Physics, Universidad Austral de Chile, Valdivia, Chile
}

\begin{abstract}
We analyze double Higgs boson production at the LHC in the context of Little Higgs models. In double Higgs production, the diagrams involved are directly related to those that cause the cancellation of the quadratic divergence of the Higgs self-energy, so this mode provides a robust prediction for this class of models. We find that in extensions of this model with the inclusion of a socalled T-parity, there is a significant enhancement in the cross sections as compared to the Standard Model.
\end{abstract}

Keywords: Higgs boson, Little Higgs Model

PACS: 123.456

The presence of quadratic divergences in loop corrections to the scalar Higgs boson self-energy is responsible for the so-called hierarchy problem of the Standard Model (SM); namely, there is no natural way of having a "light" mass (i.e. $\sim 10^{2} \mathrm{GeV}$ ) for the Higgs given that loop corrections induce mass terms of the order of the scale at which new physics enters- be it the GUT scale or any other above a few TeV. In Supersymmetric extensions this problem is absent since the divergence in bosons and fermions are related and the latter can only be logarithmic.[1]. It is also absent in models where scalar particles are not fundamental but composite[2].

Recently a new kind, called Little Higgs (LH) model [3], which can solve the hierarchy problem was proposed. Here the Higgs is a pseudo-Goldstone boson whose mass is protected by a global symmetry and, unlike supersymmetry, quadratic divergence cancellations are due to contributions from new particles with the same spin.

The phenomenology of these models has been discussed with respect to indirect effects on precision measurements [4] and direct production of the new particles introduced [5]. Since these early contributions, several variations have been proposed [6], but the cancellation of quadratic divergences is inherent to any $\mathrm{LH}$ model and this requires definite relations among certain couplings. Therefore, any process that involves exclusively these couplings is a robust prediction of the LH mechanism regardless of model variations. One of such processes is double Higgs production, which we study here.

After the spontaneous breakdown of a global underlying symmetry at a scale $4 \pi f$ of a few $\mathrm{TeV}$, the model contains a large multiplet of pseudo-Goldstone bosons, which includes the SM Higgs doublet. While most members of the multiplet receive large masses (of a few $\mathrm{TeV}$ ), the mass of the Higgs boson is protected from quadratic divergences at one loop, and therefore remains naturally smaller. The cancellation is related to the existence of an extra (heavier) top-like quark and its interactions with the scalar sector, feature 
which is common to all Little Higgs models. Higgs pair production at LHC is based on exactly the same diagrams that enter the quadratic divergence cancellation (Fig. 1), except for the insertion of two gluons (Fig. 2). In order to work out the details, we make use of the Littlest Higgs model, which is a simple case but contains all the necessary features.

Below the scale $4 \pi f$, the Little Higgs lagrangian [7] can be written as a non-linear sigma model based on a coset $S U(5) / S O(5)$ symmetry:

$$
\mathcal{L}_{\Sigma}=\frac{1}{2} \frac{f^{2}}{4} \operatorname{Tr}\left|\mathcal{D}_{\mu} \Sigma\right|^{2}
$$

where the subgroup $[S U(2) \times U(1)]^{2}$ of $S U(5)$ is promoted to a local gauge symmetry. The covariant derivative is defined as $\mathcal{D}_{\mu} \Sigma=\partial_{\mu} \Sigma-i \sum_{j=1}^{2}\left(g_{j}\left(W_{j} \Sigma+\Sigma W_{j}^{T}\right)+\right.$ $\left.g_{j}^{\prime}\left(B_{j} \Sigma+\Sigma B_{j}^{T}\right)\right)$. To exhibit the interactions, one can expand $\Sigma$ in powers of $1 / f$ around its vacuum expectation value $\Sigma_{0}$

$$
\Sigma=\Sigma_{0}+\frac{2 i}{f}\left(\begin{array}{ccc}
\phi^{\dagger} & \frac{h^{\dagger}}{\sqrt{2}} & \mathbf{0}_{2 \times 2} \\
\frac{h^{*}}{\sqrt{2}} & 0 & \frac{h}{\sqrt{2}} \\
\mathbf{0}_{2 \times 2} & \frac{h^{T}}{\sqrt{2}} & \phi
\end{array}\right)+\mathcal{O}\left(\frac{1}{f^{2}}\right)
$$

where $h$ is the doublet that will remain light and $\phi$ is a triplet under the unbroken $S U(2)$. The non-zero v.e.v. $\Sigma_{0}$ of the field leads to the breaking of global $S U(5)$ into $S O(5)$ and also breaks the gauge symmetry $[S U(2) \times U(1)]^{2}$ into its diagonal subgroup, which is identified with the standard model $S U_{L}(2) \times U_{Y}(1)$ symmetry group.

The standard model fermions acquire their masses via the usual Yukawa interactions. However, in order to cancel the top quark quadratic contribution to the Higgs self-energy, a new vector-like color triplet fermion pair, $\tilde{t}$ and $\tilde{t}^{\prime c}$, with quantum numbers $(\mathbf{3}, \mathbf{1})_{Y_{i}}$ and $(\overline{\mathbf{3}}, \mathbf{1})_{-Y_{i}}$ must be introduced. Since they are vector-like, they are allowed to have a bare mass term which is chosen such as to cancel the quadratic divergence above scale $f$. Accordingly, the standard top quark couples to the pseudo-Goldstone bosons and heavy colored fermions in the littlest Higgs model as:

$$
\mathcal{L}_{Y}=\frac{1}{2} \lambda_{1} f \epsilon_{i j k} \epsilon_{x y} \chi_{i} \Sigma_{j x} \Sigma_{k y} u_{3}^{\prime c}+\lambda_{2} f \tilde{t} \tilde{t}^{\prime c}+\text { h.c. }
$$

where $\chi_{i}=\left(b_{3}, t_{3}, \tilde{t}\right), \epsilon_{i j k}$ and $\epsilon_{x y}$ are antisymmetric tensors, and $\lambda_{1}, \lambda_{2}$ are parameters of order unity.

After EWSB, we write $h^{0}=1 / \sqrt{2}(v+H)$, and follow Perelstein et al. [8] in defining left handed fields $t_{3 L} \equiv t_{3}, \tilde{t}_{L} \equiv \tilde{t}$ and right handed fields $\bar{u}_{3 R}^{\prime} \equiv u_{3}^{\prime c}, \overline{\tilde{t}}_{R}^{\prime} \equiv \tilde{t}^{\prime c}$ to obtain

$$
\begin{aligned}
\mathcal{L}_{t}= & -\left(\begin{array}{cc}
\bar{u}_{3 R}^{\prime} & \overline{\tilde{t}}_{R}^{\prime}
\end{array}\right)\left(\begin{array}{cc}
\lambda_{1} v & \lambda_{1} f\left(1-v^{2} / f^{2}\right) \\
0 & \lambda_{2} f
\end{array}\right)\left(\begin{array}{c}
t_{3 L} \\
\tilde{t}_{L}
\end{array}\right)-\lambda_{1} H \bar{u}_{3 R}^{\prime} t_{3 L}+ \\
& \lambda_{1} \frac{v}{f} H \bar{u}_{3 R}^{\prime} \tilde{t}_{L}+\frac{\lambda_{1}}{2 f} H^{2} \bar{u}_{3 R}^{\prime} \tilde{t}_{L}+h . c .
\end{aligned}
$$




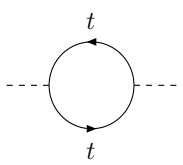

(a)

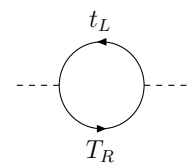

(b)

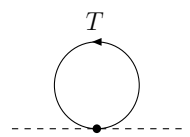

(c)

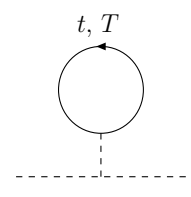

(d)

FIGURE 1. One-loop corrections to the Higgs mass, to order $v / f$ : (a) standard top quark loop, (b) mixture of standard and extra top quark loop, (c) extra top quark loop with a 4-particle vertex, and (d) tadpoles with standard and with extra top quark loops. There are other diagrams but they are suppressed by factors of order $(v / f)^{2}$ or higher.

Diagonalizing this mass matrix, we obtain the usual eigenvalues corresponding to the top quark $t$ and the heavy top $T$ masses, $m_{t}$ and $m_{T}$, in terms of the scales $v$ and $f$, and the couplings $\lambda_{i}$. From this analysis one also derives the couplings of the Higgs to the top quarks $t_{L, R}$ and $T_{L, R}$ (of left and right chirality), in terms of the same parameters. In an obvious notation, these couplings are denoted as $g_{H t t}, g_{H T_{R} t_{L}}, g_{H t_{R} T_{L}}, g_{H T T}, g_{H H T T}$ and $g_{H H t t}$. One should be aware that, for real values of $\lambda_{i}$, the values of $m_{t}, m_{T}, v$ and $f$ not only are related but also restricted to the condition: [9]

$$
m_{T}>2 \frac{m_{t} v}{f} \simeq \sqrt{2} f .
$$

The relevant Feynman diagrams for the Higgs self-energy are shown in Fig. 1.

The cancellation of tadpole diagrams requires that $g_{H t t} m_{t}+g_{H T T} m_{T}=0$, whereas the cancellation of higgs self-energy quadratic divergences implies $g_{H t t}^{2}+g_{H T T}^{2}+g_{H T_{R} t_{L}}^{2}+$ $g_{H t_{R} T_{L}}^{2}+g_{H H t t} m_{t}+g_{H H T T} m_{T}=0$. These conditions are satisfied up to terms of order $\mathcal{O}(v / f)$ by the masses and couplings defined above.

An important point to consider is that in the simplest LH models, strict bounds on the parameters exist. In particular, electroweak precision constraints require $f>3.5 \mathrm{TeV}$ [4]. However, in a recent variation on the littlest Higgs model, where a so-called T-parity that interchanges the two subgroups $[S U(2) \times U(1)]_{1}$ and $[S U(2) \times U(1)]_{2}$ of $S U(5)$ is introduced, this bound can be significantly lowered to $f>500 \mathrm{GeV}$ [14]. In this model, the T-odd states do not participate in the cancellation of quadratic divergences and in this respect our calculation is valid in this model as well. T-parity also forbids the generation of a vacuum expectation value for the triplet scalar field (i.e., $v^{\prime}=0$ in the notation of T. Han et al.[5]), which is one of the causes for easing the electroweak constraints.

Gluon-gluon fusion is the dominant mechanism of SM Higgs boson pair production at the LHC [10]. The amplitude for $g g \rightarrow H H$ process has contributions from triangle and box diagrams [9], shown in Fig. 2. All these diagrams involve integrals that can be converted to Passarino-Veltmann functions, for which there are computer codes to evaluate them. Here the expressions for the amplitudes in terms of Passarino-Veltman functions were computed using the package FeynCalc [11] and the numerical integration of these functions is done using LoopTools [12]. 


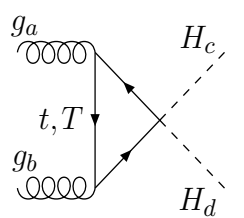

(a)

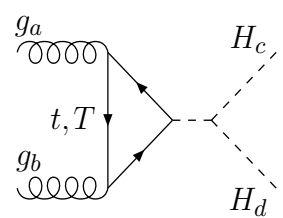

(b)

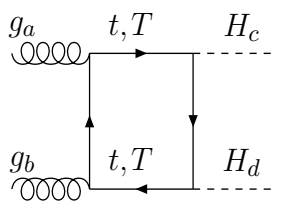

(c)

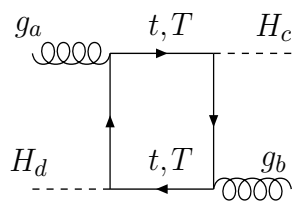

(d)

FIGURE 2. Contributions to Higgs boson pair production at LHC in a Little Higgs model: (a) and (b) triangles; (c) planar boxes; (d) non-planar boxes.

From the sum of all these diagrams and squaring, the partonic differential cross section is obtained (we have included a factor of $1 / 2$ from identical particles in the final state)

$$
\frac{d \hat{\sigma}}{d \Omega}=\frac{1}{128 \pi^{2} \hat{s}} \sqrt{1-4 M_{H}^{2} / \hat{s}} \overline{|\mathcal{M}|^{2}}
$$

where $\overline{\mid \mathcal{M}}^{2}$ is averaged over all 32 initial color and helicity states. The $p p \rightarrow H H$ cross section at LHC is then obtained by convoluting this partonic cross section with the gluon distribution functions:

$$
\begin{gathered}
\sigma(p p \rightarrow H H)=\frac{1}{2} \int d x_{1} d x_{2}\left[g_{1}\left(x_{1}, Q^{2}\right) g_{2}\left(x_{2}, Q^{2}\right)+g_{2}\left(x_{1}, Q^{2}\right) g_{1}\left(x_{2}, Q^{2}\right)\right] \\
\hat{\sigma}(g g \rightarrow H H) \theta\left(x_{1} x_{2} s-4 M_{H}^{2}\right) .
\end{gathered}
$$

Here we used the Set 3 of CTEQ6 Leading Gluon Distribution Function with momentum scale $Q^{2}=\hat{s}$ [13]. A $K=2$ factor was included to take into account QCD corrections.

In Fig. 3 we plot the cross section for the double Higgs production process at the LHC for fixed $M_{T}=4 \mathrm{TeV}$, a Higgs boson mass in the range $150-300 \mathrm{GeV}$ and for $f=500$, 1000 and $2000 \mathrm{GeV}$. As expected, we find that the largest deviations from the SM result occurs for small Higgs boson mass and small decay constant $f$.

We also explored the dependence of this cross section on the mass of the heavy top quark and found that it slowly grows with $m_{T}$, but promptly reaches an asymptotic value, becoming insensitive for $m_{T}>2.5 \mathrm{TeV}$.

\section{CONCLUSIONS}

Double Higgs production distinguishes Little Higgs (LH) models from other electroweak symmetry breaking scenarios. The process is intimately tied to the cancellation of quadratic divergences in these models. We studied the reach of the LHC to probe the LH models in this way. We found that only for relatively small values of the energy scale $f$, of the order of 500 to $1000 \mathrm{GeV}$, it is possible to distinguish meaningfully the LH from the SM. These low values are attainable without violating electroweak precision limits only in models where an extra T parity is incorporated [14]. These results are only mildly dependent on the heavy top quark mass $m_{T}$. 


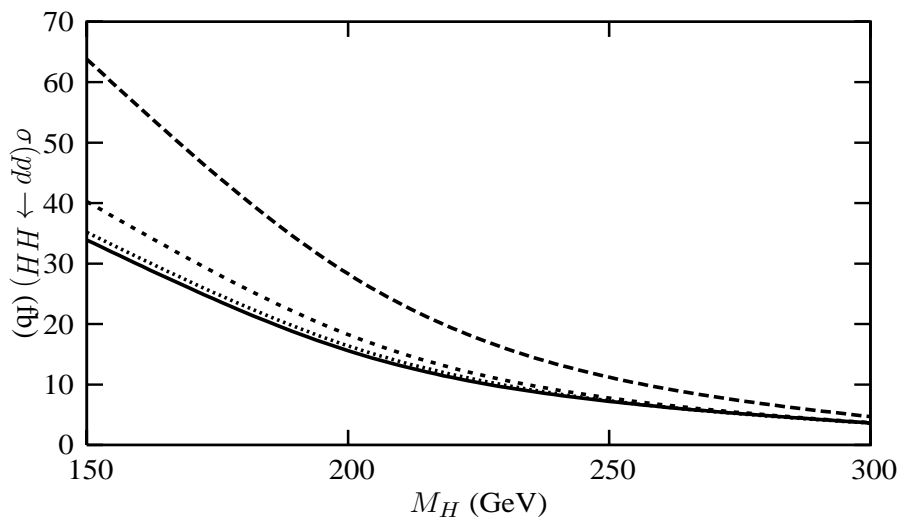

FIGURE 3. Cross section for double Higgs production at the LHC for $M_{T}=4 \mathrm{TeV}$ and $f=500 \mathrm{GeV}$ (dashed line), $1000 \mathrm{GeV}$ (short dashed line) and $2000 \mathrm{GeV}$ (dotted line). In solid line is shown the SM result.

\section{ACKNOWLEDGEMENTS}

C.D. and R.R. thank the organizing committee of this conference for the warm hospitality. A.Z. and C.D. received partial support from Fondecyt (Chile) grants No. 3020002, 1030254, 7030107 and 7040059. R.R. would like to thank CNPq for partial financial support.

\section{REFERENCES}

1. See e.g., S.P. Martin in "Perspectives in Supersymmetry," edited by G.L. Kane, World Scientific [arXiv:hep-ph/9709356].

2. For a recent review, see C.T. Hill and E.H. Simmons, Phys. Rept. 381, 235 (2003).

3. N. Arkani-Hamed, A.G. Cohen and H. Georgi, Phys. Lett. B 513, 232 (2001); N. Arkani-Hamed, A.G. Cohen, T. Gregoire and J.G. Wacker, JHEP 0208, 020 (2002); N. Arkani-Hamed, A.G. Cohen, E. Katz, A.E. Nelson, T. Gregoire and J.G. Wacker, JHEP 0208, 021 (2002).

4. C. Csaki, J. Hubisz, G.D. Kribs and P. Meade, Phys. Rev. D67, 115002 (2003); Phys. Rev. D68, 035009 (2003); J.L. Hewett, F.J. Petriello and T.G. Rizzo, JHEP 0310, 062 (2003); M.C. Chen and S. Dawson, Phys. Rev. D70, 015003 (2004).

5. G. Burdman, M. Perelstein and A. Pierce, Phys. Rev. Lett. 90, 241802 (2003) and Erratum-ibid. 92, 049903 (2004); C. Dib, R. Rosenfeld and A. Zerwekh [ArXiv:hep-ph/0302068] (unpublished); T. Han, H.E. Logan, B. McElrath and L.-T. Wang, Phys. Rev. D67, 095004 (2003); Phys. Lett. B563, 191 (2003) and Erratum-ibid. B603, 257 (2004).

6. For a recent review, see $e . g$., M. Schmaltz and D. Tucker-Smith, [arXiv:hep-ph/0502182].

7. N. Arkani-Hamed, A.G. Cohen, E. Katz, and A.E. Nelson, JHEP 0207, 034 (2002).

8. M. Perelstein, M.E. Peskin and A. Pierce, Phys. Rev. D69, 075002 (2004).

9. C. Dib, R. Rosenfeld and A. Zerwekh, in preparation.

10. See e.g., T. Plehn, M. Spira and P.M. Zerwas, Nucl. Phys. B479, 46 (1996) and Erratum ibid. 531, 655 (98)); A. Djouadi, W. Killian, M. Muhlleitner and P. M. Zerwas, Eur. Phys. J. C10, 45 (1999).

11. R. Mertig, M. Böhm and A. Denner, Comp.Phys. Commun. 64, 345 (1991).

12. T. Hahn and M. Perez-Victoria, Comput. Phys. Commun. 118, 153 (1999).

13. J. Pumplin et al. (CTEQ Collaboration), JHEP 0207, 012 (2002).

14. J. Hubisz, P. Meade, A. Noble and M. Perelstein, [arXiv:hep-ph/0506042]. 
Copyright of AIP Conference Proceedings is the property of American Institute of Physics and its content may not be copied or emailed to multiple sites or posted to a listserv without the copyright holder's express written permission. However, users may print, download, or email articles for individual use. 